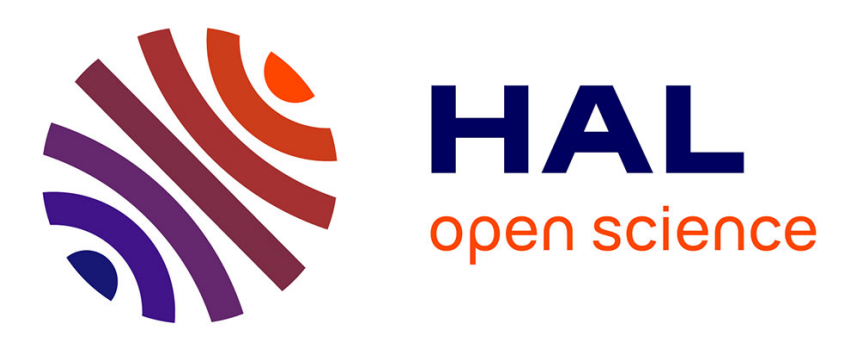

\title{
Utilisation des auxiliaires entomophages contre les ravageurs des cultures en Chine Shi-Bang Qiu
}

\section{To cite this version:}

Shi-Bang Qiu. Utilisation des auxiliaires entomophages contre les ravageurs des cultures en Chine. Agronomie, 1986, 6 (9), pp.857-861. hal-00884946

\section{HAL Id: hal-00884946 https://hal.science/hal-00884946}

Submitted on 1 Jan 1986

HAL is a multi-disciplinary open access archive for the deposit and dissemination of scientific research documents, whether they are published or not. The documents may come from teaching and research institutions in France or abroad, or from public or private research centers.
L'archive ouverte pluridisciplinaire HAL, est destinée au dépôt et à la diffusion de documents scientifiques de niveau recherche, publiés ou non, émanant des établissements d'enseignement et de recherche français ou étrangers, des laboratoires publics ou privés. 


\title{
Utilisation des auxiliaires entomophages contre les ravageurs des cultures en Chine
}

\author{
SHI-BANG QIU (*) \\ Bureau de recherche sur la protection des végétaux par moyens biologiques, Académie des Sciences Agricoles de \\ Chine (A.C.A.S.) - Beijing, Chine
}

\begin{abstract}
Bien que la Chine possède une expérience ancestrale de l'utilisation pratique des auxiliaires, des études et applications organisées de lutte biologique n'ont commencé que dans les années 1950. Durant les premières années, on a porté l'accent sur la production de masse et les lâchers de prédateurs et de parasites. Plus tard, comme l'abus des insecticides chimiques suscitait un grand nombre de problèmes nouveaux posés par les ravageurs, la sauvegarde de leurs ennemis naturels commença à retenir sérieusement l'attention et fut pratiquée à grande échelle. Des recherches sur l'aménagement de l'environnement dans le but de favoriser les ennemis naturels ont èté développées avec des résultats encourageants. Récemment l'introduction d'agents de lutte biologique en provenance de pays étrangers a connu également un certain succès. Des exemples de l'utilisation réussie d'arthropodes entomophages contre des ravageurs des cultures sont brièvement decrits. La Chine est riche de ressources en ennemis natureis, la technique des cultures intercalaires est couramment utilisée par les agriculteurs, la main-d'œuvre est disponible et relativement bon marché et le coût des pesticides est élevé. Tous ces facteurs rendent possibles la sauvegarde et l'augmentation du nombre des ennemis naturels dans de nombreux programmes de lutte contre les ravageurs.
\end{abstract}

Mots clé additionnel : Lutte biologique.

Though China has an ancestral experience in the practical use of natural enemies, the organization of studies and applications of biological control only started in the 1950s. At first, they focused on mass production and natural enemy releases. Then, as the excessive use of chemical insecticides caused many new problems with the pests, the protection of natural enemies was more thoroughly considered and was applied on a large scale. Investigations on environmental management to help natural enemies were carried out and have provided encouraging results. Recently the introduction of biological control agents from foreign countries has also been successful. Some examples of successful use of entomophagous arthropods against crop pests are briefly described. China has many resources in natural enemies, the technique of cover crops is generally used by the farmers, labour is available and relatively cheap and the cost of pesticides is high. All these factors make possible the protection and increase of natural enemies in many pest control programmes.

Additional key word : Biological control.

\section{INTRODUCTION}

Il y a maintenant environ 16 siècles qu'on recourt en Chine à l'action bénéfique des auxiliaires naturels pour détruire les insectes ravageurs des cultures. D'après les archives on sait en effet que Oecophylla smaragdina Fabr. (Hym. : Formicidae), était utilisé pour la protection des mandariniers et qu'un commerce de ces insectes était déjà pratiqué en des temps ancestraux. Aujourd'hui encore, les planteurs de la

(*) Communication présentée lors du colloque franco-chinois sur la lutte biologique, Beijing, 3-6 juin 1985. province de Guangdong emploient cet auxiliaire con: tre Rhynchocoris humeralis (Thunb.) (Heter. : Pentatomidae) et divers coléoptères (Pu, 1984). Des décrets impériaux, datant d'environ 800 ans, avaient pour objet d'assurer la protection des grenouilles qui dévorent les insectes nuisibles dans les rizières. Ce n'est pourtant qu'à partir de 1950 qu'une recherche organisée sur les ennemis naturels des ravageurs fut développée en Chine dans le but d'une utilisation rationnelle de ces auxiliaires.

Au début, ces recherches étaient limitées aux tèchniques d'élevage et de multiplication en masse. Elles ont porté par exemple sur Trichogramma sp. (Hym. : Trichogrammatidae) et Anastatus sp. (Hym. : Eupelmi- 
dae) parasites de Eucosma schistaceana Snellen (Lepid. : Tortricidae) et de Tessaratoma papillosa Drury (Heter.: Pentatomidae), ainsi que sur Dibrachys cavus Walker (Hym. : Pteromalidae), parasite de Pectinophora gossypiella Saunders (Lepid. : Gelechiidae). Ultérieurement, l'usage croissant des insecticides chimiques de synthèse à large spectre d'activité a provoqué indifféremment la destruction des ravageurs et de leurs auxiliaires, entraînant finalement un accroissement des dégâts occasionnés aux cultures. C'est pourquoi les recherches furent alors orientées sur l'utilisation rationnelle des insecticides chimiques et sur la sauvegarde des auxiliaires. Aujourd'hui la situation s'est sensiblement améliorée grâce à un meilleur respect de l'environnement écologique, assurant ainsi des conditions plus favorables à la persistance et à la multiplication des auxiliaires. Des études sont poursuivies pour accroître les niveaux de leurs populations et leur efficacité ; le concept d'introduction d'auxiliaires exotiques, jadis insuffisamment pris en compte, a été exploité de nouveau à partir de la fin des années 1970, à la faveur des relations scientifiques régulières établies avec de nombreux pays étrangers.

Ainsi, la protection des cultures par des moyens biologiques a fait de rapides progrès : elle couvrait, en 1982, 161 millions de $\mathrm{mu}$ (un $\mathrm{mu}=1 / 15 \mathrm{ha}$ ) soit près de 11 millions d'ha) contre seulement 1,2 millions de mu en 1972. En 1983, 8 millions de mu cultivés en riz, blé et coton faisaient l'objet d'une protection biologique à l'aide d'insectes utiles.

\section{MULTIPLICATION DE MASSE ET UTILISATION D'AUXILIAIRES ENTOMOPHAGES DE RAVAGEURS INDIGÈNES}

Les trichogrammes sont de loin les parasites les plus multipliés et les plus utilisés en Chine. Cinq espèces sont principalement exploitées, $T$. dendrolimi Matsum., T. confusum Vigg., T. evanescens Westw., $T$. ostriniae Pang \& Chen et $T$. japonicum Ashm., la première étant la plus commune, pour lutter contre des lépidoptères ravageurs appartenant notamment aux :

- Pyralidae: Ostrinia furnacalis Guén., Cnapha locrocis medinalis Guén., Chilo suppressalis Walk.;

- Noctuidae: Heliothis armigera Hbn., Agrotis ypsilon $\mathrm{Hfn}$.;

- Tortricidae : Eucosma schistaceana, Choristoneura longicellana Wals. :

\section{- Lasiocampidae : Dendrolimus sp.}

Ces trichogrammes sont multipliés dans les œufs de Antherea pernyi G-M (Lepid. : Saturnidae), de Philosamia cynthia ricini Bdv. (Lepid. : Saturnidae) ou de Corcyra cephalonica (Staint.) (Lepid. : Pyralidae) et non de Sitotroga cerealella (Oliv.) (Lepid. : Gelechiidae) comme dans de nombreux autres pays. Les élevages sont mécanisés pour faciliter leur mise en œuvre et assurer de hauts rendements. Récemment, l'élevage in vitro de Trichogramma sp. a été obtenu sur plus de 60 générations successives et a permis d'obtenir des résultats satisfaisants au champ avec $T$. den- drolimi et $T$. confusum (Anonyme, 1985 ; LiU et al., 1985).

S'il est d'usage courant d'employer les trichogrammes au champ sous la forme de lâchers inondatifs au moment où il convient de lutter contre les pullulations de ravageurs, cette technique met en œuvre de grandes quantités d'auxiliaires et est coûteuse. Ainsi dans la province de Shanxi, où on utilisait $T$. dendrolimi contre $H$. armigera dans les champs de cotonnier, l'efficacité du procédé restait toujours dépendante de la quantité d'auxiliaires répandus. Cependant on s'est rendu compte que l'utilisation de ces mêmes trichogrammes contre $A$. ypsilon dans les cultures maraîchères voisines de celles de cotonnier permet une multiplication suffisante de cet auxiliaire pour qu'il soit capable d'assurer aussi la protection de cette culture contre $H$. armigera et Anomis flava Fab. (Lepid. : Noctuidae). Pour un mu de culture maraîchère traitée par Trichogramma, 50 à 80 mu de culture cotonnière sont ainsi également protégés contre les attaques des noctuelles (WANG et al., 1985).

Outre les trichogrammes, les Anastatus et les Amblyseius font également l'objet d'élevages importants. La technique d'élevage des Anastatus prédateurs de T. papillosa a été améliorée (LIU et al., 1986). Dans la province du Sihuan, on a recours au pollen de diverses plantes telles que Quercus acutissima Thunb., Luffa cylindrica Roem., Brassica chinensis L.V. et Ricinus communis L. pour élever Amblyseius nicholsi Ehra \& Lee (Acar.: Phytoseiidae), prédateur de Panonychus citri (Mc Gregor) (Acar. : Tetranychidae) ravageur des mandariniers. Alors qu'une unité d'élevage donne un rendement quotidien d'environ $30000 \mathrm{~A}$. nicholsi, l'application de 300 à 600 de ces auxiliaires sur chaque mandarinier permet de protéger la culture contre les dégâts occasionnés par $P$. citri. Dans une autre province, celle de Guangdong, Amblyseius newsami (Evans) (Acar. : Phytoseiidae) est élevé sur pollen de théier, de Luffa cylindrica ou de maïs pour être utilisé également contre $P$. citri (MAI et al., 1984 ; ZHANG, 1984).

Coccinelles et chrysopes font également l'objet de recherches pour la mise au point de techniques d'élevage de prédateurs. Ainsi un aliment semi-synthétique est proposé pour l'élevage de Coccinella septempunctata L. (Col. : Coccinellidae) utilisée contre les vers du coton (CHEN et al., 1984). Alors que, dans la province du Sichuan, on recueillait les cocons de Chrysopa sinica Tjeder (PENG, 1985) pour obtenir imagos et œufs au laboratoire en vue de protéger les mandariniers contre $P$. citri, on a réussi à mettre au point la technique d'élevage des larves alimentées par des œufs de C. cephalonica (Anonyme, 1975 ; Anonyme, 1977) et celle des adultes nourris avec un milieu nutritif à base de levure de bière (ZHOU et al., 1981). L'élevage de Chrysopa sur œufs artificiels a été également réussi, sans manifestation de dégénérescence après 10 générations successives (ZHOU et al., 1985).

La multiplication en masse des auxiliaires, Encarsia formosa Gahan (Hym. : Aphelinidae) et Phytoseiulus persimilis Athias-Henriot (Acar.: Phytoseiidae) est maintenant obtenue au laboratoire dans le but de leur utilisation contre Trialeurodes vaporariorum (Westw.), (Homop. : Aleyrodidae) et Tetranychus urticae Koch. (Acar. : Tetranychidae). 


\section{SAUVEGARDE ET UTILISATION DES AUXILIAIRES NATURELS}

Bien que la méthode de multiplication en masse des auxiliaires et de lâchers inondatifs dans les champs cultivés se soit avérée fructueuse, son application reste limitée du fait que le nombre d'espèces d'auxiliaires dont on maîtrise bien la biologie et l'élevage est réduit et que les techniques d'élevage de masse sont complexes et impliquent des structures d'exploitation et du personnel spécialisé.

A partir des enquêtes faunistiques sur les ennemis naturels des ravageurs réalisées ces dernières années, on a découvert que les ressources chinoises en espèces entomophages sont extrêmement abondantes et que les insectes auxiliaires jouent un rôle très important dans le contrôle des populations de ravageurs. C'est pourquoi il a été officiellement décidé de mettre aussi l'accent sur la sauvegarde et l'utilisation des insectes utiles. Il est entendu par là la mise en ouvre de mesures appropriées pour éviter et réduire leur destruction ainsi que pour créer des conditions favorables à leur existence et à leur reproduction.

Tout comme les autres pays, la Chine a trop compté sur les insecticides chimiques pour éliminer les ravageurs, ce qui a provoqué une énorme perte d'insectes utiles. Cependant, depuis que les nouvelles mesures sont appliquées, la quantité d'insecticides épandue a considérablement régressé dans certaines régions, si bien que le rôle frénateur des auxiliaires se fait de plus en plus sentir. Ainsi, dans le district de Xiangyin, province du Hunan, après la mise en application, il y a 3 ans, des mesures visant à la protection des araignées pour prévenir les pullulations de ravageurs dans les rizières, la quantité d'insecticides employée a régressé, chaque année de 30 p. 100, tandis que dans le même temps les dégâts occasionnés par les ravageurs phytophages ont été moins importants que d'ordinaire.

En plus de ces efforts visant à réduire et à éviter les effets des insecticides chimiques sur les insectes utiles, la Chine attache aussi une grande importance à la transformation de l'environnement écologique des cultures afin d'accroître les populations et l'action limitante des auxiliaires naturels. Il est connu, par exemple, que les rizières subissent des traitements insecticides intensifs. Cet abus des interventions chimiques a gravement porté atteinte à la faune utile et a aggravé les dégâts occasionnés par des ravageurs tels que Nilaparvata lugens (Stål) (Homop. : Delphacidae) et Nephotettix sp. (Homop. : Cicadellidae). Ayant constaté que de nombreuses techniques agricoles sont aussi susceptibles d'être défavorables au développement des populations d'araignées, les scientifiques du Hunan ont préconisé l'ensemble de mesures suivantes :

1) faire des cultures hivernales afin d'offrir des gites d'hivernation ;

2) placer des gerbes de plantes herbacées au bord des rizières pendant les périodes d'irrigation ;

3) cultiver du soja sur les diguettes afin d'offrir aux araignées un abri et un terrain de chasse complémentaire ;

4) utiliser les insecticides d'une façon plus rationnelle et opportune.
Ces expériences ont été couronnées de succès et elles sont maintenant mises en pratique sur plus de $18 \mathrm{mil}$ lions de mu dans cette province du Hunan (ANONYME, 1984).

Un autre exemple est donné par la stratégie d'utilisation des coccinelles pour lutter contre les pucerons : autrefois, on mobilisait les écoliers pour récolter dans les champs de blé les coccinelles qui ensuite étaient dispersées dans les champs de cotonnier. Les résultats étaient satisfaisants mais nécessitaient le recours à une très forte main-d'œuvre dont l'activité était d'ailleurs plus ou moins préjudiciable à la récolte de blé. Puis on se mit à intercaler cotonnier et blé ou cotonnier et colza. Les ravageurs du blé ou du colza attirent des auxiliaires, des prédateurs comme la coccinelle à 7 points, qui se multiplient et passent sur le cotonnier lorsque le blé mûrit ou bien lorsque après la récolte du colza, on en utilise les tiges comme engrais vert.

En Chine du nord, on cultive principalement le blé, le maïs et le cotonnier. Au début du printemps, les prédateurs tels que Chrysopa et Orius sont présents dans les champs de blé. Au lieu d'utiliser des insecticides chimiques non sélectifs pour protéger la céréale des pullulations de Mythimna separata Walk. (Lepid. : Noctuidae), on emploie maintenant le «Chitin forming inhibitor " (ANONYME, 1981); dans les champs de cotonnier on remplace la pulvérisation du feuillage par une application de granulés localisée à proximité des racines, sans danger pour les prédateurs.

Dans les vergers des principes analogues peuvent aussi être appliqués. Ainsi dans la province de Guangdong, où les fortes températures et l'absence de brume ou de rosée sont défavorables à Amblyseius newsami prédateur de $P$. citri, on cultive Ageratum conyzoides comme couverture végétale du sol entre les mandariniers de façon à abaisser la température et à augmenter l'humidité relative. $A$. conyzoides présente en outre l'avantage de fournir un pollen que consomme A. newsami et d'être éventuellement utilisable comme engrais vert. Dans ces conditions, le contrôle des pullulations de $P$. citri est pratiquement assuré (MAI et al., 1984).

Dans la région de Beijing, c'est la culture de la luzerne qui est préconisée dans les vergers de pommier, à la fois pour couvrir le sol et pour offrir un refuge aux auxiliaires tels que les coccinelles, syrphes et chrysopes. Dans certaines localités de la province de Jiangsu, ce sont des cultures intercalaires de Vicia villosa Roth. qui sont pratiquées dans les vergers de pommier. Après la récolte des Vicia, la faune utile des coccinelles, chrysopes et Orius minutus L. (Heter.: Anthocoridae) se déplace sur les arbres fruitiers où elle contrôle en particulier les populations de Tetranychus viennensis Zacher (QUIN, 1985).

\section{INTRODUCTION-ACCLIMATATION D'AUXILIAIRES EXOTIQUES}

La stratégie d'introduction-acclimatation a été mise en œuvre dès 1929 dans la région de Shanghai avec l'importation de Rodolia cardinalis (Muls.) (Col. : Coccinellidae) prédateur d'Icerya purchasi Mask. 
(Homop. : Margaroidea), lui-même ravageur des Pittosporum. Une opération identique fut réalisée dans les années 50 dans la province du Guangdong toujours contre I. purchasi mais cette fois sur Ephedra sinica. Des, Cryptolaemus montrouzieri (Muls.) (Col. : Coccinellidae), des Aphelinus mali (Haldem.) (Hym.: Aphelinidae) et des Lixophaga diatraeae Townsend (Dipt. : Tachinidae) furent également importés pour lutter respectivement contre Pseudococcus spp. (Homop.: Lecanoidea), Eriosoma lanigerum (Hausm.) (Homop. : Phemphigidae) et Diatraea saccharalis (Fabr.) (Lepid. : Pyralidae). Parmi tous ces auxiliaires, c'est la coccinelle $R$. cardinalis qui s'est avérée la plus performante ; elle a été acclimatée dans d'autres provinces et régions autonomes de Chine, et notamment au Guangsei, au Zhejiang, au Jiangsu, au Sihuan et au Hebei. On a aussi constaté que le croisement des Aphelinus mali introduits avec les souches locales de Qingdao a provoqué un renforcement de la vigueur et de l'activité parasitaire de cet auxiliaire naturel.

C'est à la fin des années 1970 que la Chine a institué un organisme officiel chargé de l'introduction et des échanges d'auxiliaires avec l'étranger. Au cours des 5 à 6 dernières années, les échanges se sont multipliés avec une dizaine de pays :

- importation de Encarsia formosa depuis la Grande-Bretagne et de Phytoseiulus persimilis depuis le Chili pour lutter contre les ravageurs des cultures sous verre ;

- introduction de Metaseiulus occidentalis Nesbitt (Acar. : Phytoseiidae), résistant aux insecticides, en provenance des Etats-Unis pour contrôler les acariens phytophages ravageurs des pommiers ;

- premiers succès de l'utilisation du nématode entomophage Neoaplectana fletiae Weiser, introduit d'Australie, contre Carposina nipponensis Walsingham (Lepid. : Carposinidae).

C'est seulement à partir de 1981 que les premiers échanges de matériel biologique avec la France ont été établis.

A l'intérieur même de la Chine, le transfert de populations d'insectes utiles paraît également très pro- metteur. C'est ainsi qu'entre 1953 et 1954 , on a introduit avec succès la coccinelle Rodolia rufopilosa (Muls.) (Col. : Coccinellidae) dans le Sihuan à partir des populations présentes dans le Zhejiang et le Hubei pour lutter contre Icerya purchasi.

De même, on a procédé en 1977 à l'introduction dans le district de Fengshun, province du Guangdong, de l'entomophage Bracon greeni Ashm. (Hym. : Braconidae), originaire de l'île de Hainan, pour limiter les populations du prédateur Eublema amabilis Moore (Lepid. : Noctuidae) qui s'attaque à Laccifer lacca (Kerr.) (Homop. : Lecanoidea) producteur de laque (PU, 1984).

\section{CONCLUSION}

Ces différents exemples ne sont qu'une illustration de l'utilisation des insectes entomophages en Chine. C'est en 1975 que la Chine a affirmé le principe " d'une protection des végétaux mettant l'accent sur la prévention et le contrôle des maladies et des ravageurs d'une façon intégrée ". Depuis lors, une importance plus grande encore a été accordée aux moyens biologiques de lutte.

La Chine est un pays très vaste où abondent à la fois une main-d'œuvre bon marché et des ressources importantes en insectes entomophages. Les paysans y pratiquent une agriculture intensive utilisant une grande diversité de systèmes culturaux et le coût des insecticides chimiques y est assez élevé. C'est pourquoi d'excellentes conditions sont réunies pour favoriser la multiplication en masse des auxiliaires et leur sauvegarde dans la nature. Si les cultures sont prometteuses, l'état actuel de nos connaissances ne permet pas encore de répondre à tous nos besoins. Il est donc souhaitable d'accroître les échanges et de développer encore davantage les collaborations entre les chercheurs chinois et leurs collègues étrangers.

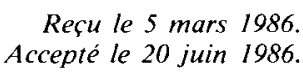

\section{RÉFÉRENCES BIBLIOGRAPHIQUES}

Anonyme, 1975. Studies on the collective rearing of Chrysopa larvae. Kunchong Zhishi., 12 (4).

Anonyme, 1977. Rearing Chrysopa sinica in winter. Kunchong Zhishi., 14 (5), 143-144.

Anonyme, 1981. Control Mythimna separata with two chitinforming inhibitors. Plant Prot., 7 (1), 26-27.

Anonyme, 1984. Protection and utilization of spiders in rice fields, p. 66-83. In " General Station of Plant Protection, Ministry of Agriculture, Animal Husbandry and Fishery. Advance in biological control in China." Agriculture Press, Beijing, 437 p.

Anonyme, 1985. Study on artificial host eff-EII for Trichogramma. J. Wuhan Univ. (Natural Science Edition), 4, 1-10.

Chen Z. H., Qin J. D., Fan X. N., Li X. L., 1984. Effects of adding lipids and juvenoid into the artificial diet on feeding and reproduction of Coccinella septempunctata L., Acta entomol. sin., 27 (2), 136-146.

Liu Z. C., Sun S. R., Wang Z. Y., Liu J. F., Zhang Q. X., Dai K. J.,
Gao Y. G., 1985. Field release of Trichogramma confusum reared on artificial host eggs against sugarcane borers. Chin J. biol. Control, 1 (3), 2-5.

Liu Z. C., Wang Z. Y., Sun S. R., Liu J. F., Yang W. H., 1986. Mass propagation of Anastatus sp., a parasitoid of Litchi stink bug with artificial host egg. Chin. J. biol. Control, 2 (2), 5-8.

Mai X. H., Huang M. D., Li S. X., Xong J. J., 1984. Utilization and protection of predacious mites for citrus mite control, p. 310326. In " General Station of Plant Protection, Ministry of Agriculture, Animal Husbandry and Fishery. Advance in biological control in China. " Agriculture Press, Beijing, 437 p.

Peng Y. K., 1985. Field release of Chrysopa sinica as a strategy in the integrated control of Panonychus citri. Chin. J. biol. Control, 1 (1), 2-7.

Pu Z. L., 1984. Principles and methods of biological control of insect pests (2nd ed.). Science Press, Beijing, $318 \mathrm{p}$.

Quin S. H., 1985. Field rearing of predacious mites for the control of apple orchard mites. Enemies Insects, 7 (2), 1-8. 
Wang F. G., Zhang S. Y., Hou S. R., 1985. Inoculative release of Trichogramma dendrolimi in vegetable gardens to regulate populations of cotton pests. Chin. J. biol. Control, 1 (4), 2-7.

Zhang G. C., 1984. Control citrus leaf mite with predacious mites, p. 278-309. In " General Station of Plant Protection, Ministry of Agriculture, Animal Husbandry and Fishery. Advance in biological control in China." Agriculture Press, Beijing, 437 p.
Zhou W. R., Liu Z. L., Cheng W., Qiu S. B.. 1981. An artificial dry powder diet for adults of Chrysopa sinica. Plant Prot., 7 (5), 2 3 .

Zhou W. R., Chen H. Y., Qiu S. B., 1985. Rearing Chrysopa sinica on a simplified encapsulated diet for ten successive generations. Chin. J. biol. Control, 1 (1), 8-11. 